\title{
¿Es necesaria la profilaxis antibiótica en niños con reflujo vesicoureteral leve-moderado, grado II-III?
}

\author{
M. Aparicio Rodrigo \\ Pediatra. CS Entrevías. Servicio Madrileño de Salud, Área I. Madrid. España.
}

Rev Pediatr Aten Primaria. 2009; I 1:289-98

María Aparicio Rodrigo, maparicio.gapm0I@salud.madrid.org

\section{Resumen}

Conclusiones de los autores: en la revisión sistemática concluyen que no existe suficiente evidencia para aconsejar o desaconsejar la profilaxis antibiótica (PAB) de forma generalizada a los niños con reflujo vesicoureteral (RVU). La guía de práctica clínica, con un nivel de evidencia $C$, no recomienda usar de forma generalizada $P A B$ en niños con reflujo I-III. El ensayo clínico aleatorio demuestra que la $P A B$ en niños de hasta 30 meses con RVU grados I a IV no tiene ventajas sobre no tratar.

Conclusiones de los revisores: en vista de la evidencia disponible, no sería necesario administrar PBA a niños menores de 30 meses con RVU grados I a IV. Aunque no está claro el papel de las infecciones urinarias sobre la progresión del daño renal en niños con RVU parece razonable mantener una actitud de vigilancia ante posibles síntomas de infección urinaria para realizar un cultivo e iniciar de forma precoz el tratamiento antibiótico adecuado.

Palabras clave: Infección del tracto urinario, Reflujo vesicoureteral, Profilaxis antibiótica.

Abstract

Author's conclusions: the systematic review concludes that there is not enough evidence to recommend or not the use of antibiotic prophylaxis in children with vesicoureteral reflux. The guideline practice, with a $C$ evidence level, does not recommend antibiotic prophylaxis for children with I-III grade vesicoureteral reflux. Finally the randomized controlled trial shows that antibiotic prophylaxis in children 1-30 months with vesicoureteral reflux grade I$\mathrm{IV}$ is not effective in reducing the rate of infection or the incidence of renal damage or progression.

Reviewers' commentary: evidence shows that antibiotic prophylaxis in children 1-30 months old with vesicoureteral reflux grade I-IV is not effective in preventing urinary tract infections. Even though it is not clear the role of urinary infections in the progressive damage of renal parenchyma in children with vesicoureteral reflux, it seems acceptable that an early and correct diagnosis and prompt treatment remain the basis of the management of these children.

Key words: Urinary tract infection, Vesicoureteral reflux, Antibiotic prophylaxis.

Este artículo se publica simultáneamente con la revista electrónica Evidencias en Pediatría (EeP, www.aepap.org/EvidPediatr/index.htm). La autora declara no presentar conflictos de intereses en relación con la preparación y publicación de este artículo. 


\section{Escenario clínico}

Los padres de un varón de 9 meses acuden a la consulta porque su hijo ha sido diagnosticado de reflujo vesicoureteral (RVU). Tras un episodio con fiebre de $39,5^{\circ} \mathrm{C}$ hace un mes, fue ingresado en el hospital con el diagnóstico de infección del tracto urinario por $E$. coli (ITU). Un mes después del alta le hicieron nuevas pruebas radiológicas y ayer acudieron a la consulta del hospital para recoger los resultados. Les han dicho que tiene un RVU grado II sin alteraciones morfológicas renales (ecografía normal) y que debe tomar una dosis baja de antibiótico todas las noches durante unos años hasta que el RVU desaparezca. Son unos padres jóvenes, con nivel de estudios medio que desde el principio han mostrado desconfianza hacia la medicina tradicional, cuestionándose el uso de algunas vacunas.

En este caso los padres quieren que les explique qué le pasa al niño y si es necesario este tratamiento. Les indico que para tratar el tema hace falta más tiempo y les cito para informarles la semana siguiente.

\section{Pregunta clínica}

En niños menores de 24 meses con reflujo vesicoureteral, ¿la profilaxis con antibiótico resulta eficaz para disminuir las infecciones de orina o el daño renal?

Fecha de formulación: marzo de 2009.

\section{Búsqueda bibliográfica}

1. Biblioteca Cochrane Plus: estrategia de búsqueda "children and (urinary tract infection or vesicoureteral reflux)" buscando en bases de datos de revisiones sistemáticas y en la base de datos de ensayos clínicos (CENTRAL). En esta base de datos se seleccionó una revisión sistemática':

Hodson EM, Wheeler DM, Vimalchandra D, Smith GH, Craig JC. Intervenciones para el reflujo vesicoureteral primario (Revisión Cochrane traducida). En: La Biblioteca Cochrane Plus, 2008 Número 4. Oxford: Update Software Ltd. Disponible en: http://www.update-software.com. (Traducida de The Cochrane Library, 2008 Issue 3. Chichester, UK: John Wiley \& Sons, Ltd.).

2. Base de datos MEDLINE, a través de PubMed (www.pubmed.org).

Estrategia de búsqueda: descriptores "(Vesico-Ureteral Reflux [Mesh] OR Urinary Tract Infections[Mesh]) AND (Antibiotic Prophylaxis[Mesh] AND prevention and control[Subheading] ) con los limitadores: edad ("all infant:_ birth-23 months, preschool child: 2-5 years"), 
idioma ("English, French, Spanish"), tipo de estudio ("Meta-Analysis, Practice Guideline, Randomized Controlled Trial, Review") y fecha de publicación: últimos 5 años. También se realizó una búsqueda libre con los terminos: (urinary tract infection OR vesicoureteral reflux) AND antibiotic AND prophylaxis con los mismos limitadores de la búsqueda anterior. En esta base de datos se seleccionó el artículo:

Pennesi $M$, Travan $L$, Peratoner $L$, Bordugo A, Cattaneo A, Ronfani L, Minisini $S$, Ventura A; North East Italy Prophylaxis in VUR study group. Is antibiotic prophylaxis in children with vesicoureteral reflux effective in preventing pyelonephritis and renal scars? A randomized, controlled trial. Pediatrics. 2008;121:e1489-94.

3. Guíasalud, a través del portal www. guiasalud.es/egpc/index.html

Estrategia de búsqueda: reflujo vesicoureteral. Guía seleccionada?:

Areses Trapote R, Escribano Subías J, Fraga Rodríguez G, García Romero J, Loris Pablo C, Valenciano Fuente B. Guía de práctica clínica: manejo del paciente con reflujo vesicoureteral primario o esencial. Guíasalud (www.guiasalud.es/ GPC/GPC_441_Reflujo_VU.pdf).
Resumen estructurado de los artículos seleccionados

Hodson EM, Wheeler DM, Vimalchandra D, Smith GH, Craig JC. Intervenciones para el reflujo vesicoureteral primario (Revisión Cochrane traducida). En: La Biblioteca Cochrane Plus, 2008 Número 4. Oxford: Update Software Ltd. Disponible en: http://www.update-software. com. (Traducida de The Cochrane Library, 2008 Issue 3. Chichester, UK: John Wiley \& Sons, Ltd.).

Objetivo: evaluar los beneficios y daños de las diferentes opciones de tratamiento para el RVU primario.

Fuente de datos: se realizó la búsqueda en la base de datos COCHRANE: Cochrane Renal Group y en CENTRAL hasta junio de 2006; MEDLINE desde 1966 hasta febrero de 2003; EMBASE desde 1966 hasta febrero de 2003. Se utilizaron los siguientes descriptores: (vesico-ureteral reflux.sh) or (vesicoureteral reflux.tw) or (VUR.th) or (vesicoureteric reflux.tw) or vesicoureteric reflux). La búsqueda se completó con la lista de referencia de artículos, revisiones y estudios pertinentes, representantes de la industria farmacéutica y autores conocidos del tema (todos hasta febrero de 2003). 
Selección de artículos: ensayos controlados aleatorios o cuasialeatorios que considerasen cualquier tratamiento para el RVU, en población de ambos sexos y cualquier edad. Se excluyeron los pacientes con RVU asociado con válvulas uretrales posteriores, espina bífida, otras alteraciones urológicas o trasplantes renales. La calidad metodológica se evaluó mediante el método de Schulz 1995 (asignación al azar, ocultación de la asignación, cegamiento de los evaluadores del resultado, análisis por intención de tratar y porcentaje de pérdidas en el seguimiento). Se seleccionaron once ensayos y se excluyeron cuatro. Dos ensayos de los seleccionados hacen referencia a la pregunta que nos ocupa.

Extracción de datos: la realizaron dos personas de forma independiente y una tercera persona resolvió las discrepancias. Los datos se combinaron con el modelo de efectos aleatorios y los dicotómicos se presentaron como riesgo relativo e intervalo de confianza. Los datos analizados fueron: incidencia de ITU y alteraciones en el parénquima renal en pacientes con profilaxis antibiótica (PAB) continua vs. supervisión $(\mathrm{SB})$ y en pacientes con $P A B$ intermitente vs. SB.

Resultados principales: se estudió un total de 142 niños cuando se comparó la $P A B$ continua con $S B$, y 30 niños cuando se valoró la $\mathrm{PAB}$ intermitente con $\mathrm{SB}$; no encontraron diferencias significativas en el riesgo de infección urinaria (ITU) entre PAB continua vs. SB (2 estudios) (RR 0,75; intervalo de confianza del $95 \%$ [IC 95\%]: 0,15 a 3,84), PAB intermitente vs. SB (1 estudio) (RR 0,46; IC 95\%: 0,1 a 2,00). Tampoco encontraron diferencias en relación al daño renal al comparar PAB continua vs. SB (2 estudios) (RR 1,70; IC $95 \%$ : 0,36 a 8,07) ni al comparar $\mathrm{PAB}$ intermitente vs. SB (1 estudio) (RR 0,38; IC 95\%: 0,02 a 8,59).

Conclusiones de los autores: no existe certeza de que la profilaxis antibiótica de niños con RVU ofrezca un beneficio clínicamente importante.

Conflicto de intereses: no existen.

Fuente de financiación: en parte por una subvención inicial de la Fundación Renal Australiana (S2/99).

Pennesi $M$, Travan $L$, Peratoner $L$, Bordugo A, Cattaneo A, Ronfani $L$, et al; North East Italy Prophylaxis in VUR study group. Is antibiotic prophylaxis in children with vesicoureteral reflux effective in preventing pyelonephritis and renal scars? A randomized, controlled trial. Pediatrics. 2008;121:e1489-94.

Objetivo: evaluar el efecto de la PAB continua sobre la prevención de recu- 
rrencias de pielonefritis (PNA) y cicatrices renales (CR) en niños menores de 30 meses con RVU.

Diseño: ensayo clínico multicéntrico, aleatorizado y abierto (sin enmascaramiento).

Emplazamiento: siete hospitales pediátricos del norte de Italia.

Población de estudio: se seleccionó un total de 124 niños. Los criterios de inclusión fueron: presencia de RVU grado II, III o IV mono o bilateral diagnosticado entre 1 y 30 días tras un primer episodio de PNA y edad inferior a 30 meses al diagnóstico. Se definió episodio de PNA como fiebre sin foco superior a $38^{\circ} \mathrm{C}$ axilar, alteraciones en el sedimento urinario y urocultivo, recogido por bolsa estéril o cateterización, positivo para la misma bacteria en dos muestras diferentes. A estos pacientes se les realizó una ecografía, una cistouretrografía a los 2 meses y DMSA a los 6 meses. Un total de 24 pacientes rechazaron la participación en el estudio y finalmente se aleatorizaron un total de 100 niños. No hubo pérdidas en el seguimiento.

Intervención: se asignaron de forma aleatoria, estratificada (en función de la edad, sexo y grado de reflujo) a un grupo de intervención $(n=50)$, al que se administró $\mathrm{PAB}$ continua con trimetroprim (1-
$2 \mathrm{mg} / \mathrm{kg}) / \mathrm{sulfametoxazol}(5-10 \mathrm{mg} / \mathrm{kg})$ una vez al día, o a un grupo control ( $n=$ 50) que no recibió PAB. Ante un episodio febril o síntomas de infección urinaria (irritabilidad, anorexia o mal olor de orina) se realizaron un cultivo y un sedimento de orina. La PAB se mantuvo durante 2 años, fecha en la que se repitió la cistografía, ecografía y DMSA a todos los pacientes. El seguimiento se mantuvo hasta un total de 4 años, momento en que se repitió la cistografía si en la previa persistía el RVU, y el DMSA si el previo presentaba alteraciones o si fue normal pero el paciente presentó un episodio de PNA en el seguimiento y en todos los niños con alteraciones ecográficas. Los radiólogos y microbiólogos que realizaron las pruebas fueron ciegos a los grupos.

Medición de los resultados: se consideró como variable principal la recurrencia de PNA, y como variable secundaria la progresión del daño renal medida mediante DMSA. Se describieron los criterios diagnósticos para la valoración del DMSA. Se comprobó la presencia de antibiótico en orina para los niños con PNA de repetición. A las variables continuas se las aplicó un análisis de la varianza y a los datos discontinuos el test $\chi^{2}$.

Resultados principales: en el grupo de $P A B$ hubo un $36 \%$ de recurrencias 
de PNA, frente a $30 \%$ en el grupo control (RR 1,2; IC 95\%: 0,68 a 2,11). No se encontraron diferencias en la incidencia de infección según el grado de reflujo. Ambos grupos fueron similares tanto en las características basales generales demográficas, como en el grado de reflujo y presencia de cicatrices en el DMSA. La presencia de cicatrices renales al final de los 4 años de estudio fue de un $40 \%$ en el grupo con PAB y de $36 \%$ en el grupo control (RR 1,22; IC 95\%:0,75-1,98). No se detectaron alteraciones en pacientes con DMSA previo normal.

Conclusión de los autores: el uso de $P A B$ continua en pacientes con RVU, grados II a IV, no disminuye la tasa de PNA ni la incidencia de cicatrices renales o su progresión, que parece más asociada a la presencia de RVU y sus grados. Probablemente la clave de la protección renal esté en la detección y el tratamiento precoz de los episodios de PNA.

Conflictos de intereses: no existen.

Fuente de financiación: becas 35/00 del Ministerio de Sanidad italiano.
Areses Trapote R, Escribano Subías J, Fraga Rodríguez G, García Romero J, Loris Pablo C, Valenciano Fuente B. Guía de práctica clínica: manejo del paciente con reflujo vesicoureteral primario o esencial. Guíasalud. Asociación Española de Nefrología Pediátrica; 2008 [consultado el 03/06/2009]. Disponible en www.guiasalud.es/GPC/GPC_441_Re flujo_VU.pdf

Objetivo: mejora del seguimiento y tratamiento del RVU primario por parte de los pediatras de Atención Primaria y Especializada.

Fuentes de datos: búsqueda sistemática de la literatura en las bases: MEDLINE, EMBASE, LILACS_BIREME, Índice Médico Español y en las siguientes fuentes terciarias: Evidence Based Medicine, Archimedes, Evidencias en Pediatría, Colaboración Cochrane, Centro Cochrane Iberoamericano, Scottish Intercollegiate Guidelines Network (SIGN), NICE, GPC de la Nacional Guideliness Cleringhouse, GPC de la Canadian Médical Association, Guíasalud, Clinical Evidence TRIP database y SUMsearch; desde 1990 a marzo de 2008. No se describen los descriptores empleados y la búsqueda se restringió a los idiomas español, inglés y francés.

Selección de estudios: se seleccionaron por orden de prioridad revisiones sis- 
temáticas, metaanálisis, ECAs, estudios de cohortes y casos y controles. Para la elaboración de los niveles de evidencia y grado de recomendación se empleó la metodología del SIGN para las preguntas de intervención y del Centre for Evidence -Based-Medicine de Oxford (CASP) para los diagnósticos. Se seleccionó un total de 199 trabajos. Para responder a la pregunta de nuestra búsqueda se basaron en tres ECAs.

Extracción de datos: dos personas de forma independiente analizaron la evidencia mediante las plantillas CASP y los resultados se recogieron en tablas de evidencia. Las recomendaciones se resolvieron por consenso del grupo redactor. La guía fue evaluada por revisores externos.

Resultados principales: en el primer ECA $(N=43)$ se compararon PAB continua, PAB 3 días por semana y SB en niños con RVU de cualquier grado. No encontraron diferencias entre la $P A B$ continua y SB para prevenir ITU (RR 0,25; IC 95\%: 0,03 a 1,83), ni entre $P A B$ intermitente y SB (RR 0,46; IC 95\%: 0,02 a 9,18). En el segundo estudio ( $\mathrm{N}=$ 113) se realizó la comparación entre niños con RVU I a III con profilaxis AB continua frente a control sin profilaxis (SB). Tras un año de seguimiento no encontraron diferencias en el número de infecciones recurrentes (RR 1,05; IC 95\%:
0,54 a 2,07), ni en la aparición de lesiones parenquimatosas (RR 0,64; IC 95\% 0,53 a 13,03). El último ( $N=225)$ evaluó la PAB en niños hasta 3 años con RVU I a III y tampoco encontraron diferencias en la ocurrencia de ITU entre PAB y SB (17\% vs. $26 \% ; p=0,15)$.

Conclusión de los autores: en los niños con RVU primario grados I-III no se recomienda la $\mathrm{PAB}$ de forma generalizada (evidencia C).

Conflicto de intereses: todos los miembros del equipo de trabajo realizaron una declaración de intereses. No existe conflicto.

Fuente de financiación: Asociación Española de Nefrología Pediátrica, Instituto Aragonés de Ciencias de la Salud.

\section{Comentario crítico}

Justificación: el RVU primario es una patología que afecta al $1 \%$ del la población infantil, y se presenta en el 30-50\% de niños con ITU. Se define como el paso retrógrado de orina desde la vejiga al uréter sin que exista una causa anatómica que lo justifique. Aunque se ha considerado que las infecciones urinarias son la principal causa de daño permanente del parénquima renal en los niños con $R V U$, y de la progresión a insuficiencia renal crónica e hipertensión ${ }^{4}$, esto se está poniendo en duda en los últimos años ${ }^{5}$. 
Basándose en el primer supuesto, y sin una base científica clara, desde 1978 se recomienda el uso de antibióticos a largo plazo para evitar PNA en niños con $\mathrm{RVU}^{6}$. Esta práctica se está cuestionando en los últimos años, conduciendo a discrepancias en los tratamientos según los centros, más aún cuando publicaciones recientes muestran como el tratamiento tanto médico como quirúrgico a niños con RVU no se ha asociado con una disminución del fracaso renal secundario a RVU7. Todo ello justifica una revisión sobre el tema para conocer la evidencia científica que sostiene el tratamiento antibiótico a largo plazo en el RVU.

Validez o rigor científico: en la revisión Cochrane ${ }^{1}$, aunque está actualizada a 2008, las referencias bibliográficas tienen como límite junio de 2006 en el Registro Cochrane y 2003 en el resto de bases, lo que puede haber dejado fuera trabajos más actuales. Se seleccionaron dos estudios para responder a la pregunta planteada (Garin 2006: 113 niños de 3 a 12 años con RVU grados I-III, calidad baja 2/5; Reddy 1997: 43 niños sin referencia a edad ni grado de reflujo, divididos en tres grupos de estudio, calidad baja 1/5). Los estudios no fueron homogéneos y por ello es dudosa la validez del resultado global; el rango de edad (en el estudio donde se describe esta) es muy amplio, así como los intervalos de confianza. Por tanto, la fuerza de las conclusiones es débil. Tal y como indican los autores, no hay evidencia suficiente para defender la administración o no de PAB para el tratamiento del RVU.

El estudio de Pennesi ${ }^{2}$ es un ECA de alta calidad. Se calculó el tamaño de la muestra en función de la variable principal, pero pudo ser escasa para la variable secundaria. Los métodos de medición de las variables principal y secundaria fueron adecuados. El estudio no fue ciego para los pacientes ni para los profesionales que procesaron y evaluaron los datos, pero sí para los radiólogos que realizaron los DMSA y microbiólogos que analizaron las muestras de orina. Aunque las variables medidas fueron de carácter objetivo y hubo cegamiento parcial, no se puede asegurar que no pudiera existir un sesgo en la actuación de los padres y los clínicos. Los autores no indican el número de cultivos realizados, pero en el supuesto de que se hubiesen hecho más en niños control esto no se tradujo en mayor número de infecciones. Tampoco se indica el método de recogida de orina para confirmar las infecciones durante el seguimiento, pero en cualquier caso fue el mismo en los dos grupos. Hubo cuatro pacientes del grupo control y seis del de intervención que no siguieron el protocolo, aunque se realizó un análisis por intención de tratar. 
La guía de práctica clínica ${ }^{3}$ basa sus recomendaciones en tres estudios: Reddy (1997), Garin (2006) y Roussey-Kesler (2008). Los dos primeros, como ya se ha comentado en la primera referencia de esta revisión, son estudios con baja calidad metodológica cuyos resultados no presentan una evidencia suficiente para hacer una recomendación. La última referencia estudia una población similar a la de Pennesi ${ }^{2}$ pero más numerosa $(\mathrm{N}=$ 225) con reflujo menos grave (I a III). La medición de la variable principal fue poco precisa, dado que se consideró ITU positiva con un urocultivo recogido con bolsa; el seguimiento fue de 18 meses y no se hace estudio de daño renal.

Aplicabilidad a la práctica clínica: la administración de antibióticos a bajas dosis y durante un tiempo prolongado es una práctica que favorece las resistencias bacterianas. Las publicaciones más recientes coinciden en que la PAB no disminuye el número de infecciones urinarias en niños con RVU grados II, III y IV, en los primeros años de vida ( 0 a 30 meses), período en el que el riñón es más susceptible de ser dañado. Además en los casos en los que se producen ITU parece que estas no tienen relación con la aparición o empeoramiento de las cicatrices renales, aunque el estudio de Pennesi no se diseñó para analizar este aspecto y estos re- sultados deben mirarse con cautela. Todo ello sugiere que no es necesaria la PAB en niños con RVU de grado leve-moderado.

Estos hallazgos dejan la puerta abierta a nuevas investigaciones que aclaren la causa de la progresión del daño renal en niños con RVU una vez descartadas las ITU.

\section{Resolución del escenario clínico}

La semana siguiente cuando acuden los padres a la consulta, se les explica qué es el RVU y el teórico riesgo sobre el desarrollo renal; que la administración profiláctica de antibióticos a los niños con RVU se realiza desde los años 70 por un probable beneficio conceptual y en base a la investigación animal, pero no en base a la demostración de su eficacia en humanos. También se les dice que recientemente se cuenta con el resultado de estudios con buena calidad metodológica y pacientes con características similares a su hijo que demuestran que la profilaxis antibiótica no muestra ventajas sobre el no tratamiento en la prevención de las ITU. Así, si están de acuerdo, no se prescribirá el antibiótico, aunque conviene estar pendiente de cualquier signo de ITU. Ante procesos con fiebre alta sin foco se debe realizar un urocultivo para descartar ITU y poner un antibiótico adecuado de forma precoz. 


\section{Bibliografía}

1. Hodson EM, Wheeler DM, Vimalchandra $D$, Smith GH, Craig JC. Intervenciones para el reflujo vesicoureteral primario (Revisión Cochrane traducida). En: La Biblioteca Cochrane Plus, 2008 Número 4. Oxford: Update Software Ltd. Disponible en: http://www.update-software.com. (Traducida de The Cochrane Library, 2008 Issue 3. Chichester, UK: John Wiley \& Sons, Ltd.).

2. Pennesi $M$, Travan $L$, Peratoner $L$, Bordugo $A$, Cattaneo A, Ronfani $L$, et al. North East Italy Prophylaxis in VUR study group. Is antibiotic prophylaxis in children with vesicoureteral reflux effective in preventing pyelonephritis and renal scars? A randomized, controlled trial. Pediatrics. 2008;121: e1489-94.

3. Areses Trapote R, Escribano Subías J, Fraga Rodríguez G, García Romero J, Loris Pablo C, Valenciano Fuente B. Guía de práctica clínica: manejo del paciente con reflujo vesicoureteral primario o esencial. Guíasalud. Asociación Española de Nefrología Pediátrica; 2008 [consultado el 03/06/2009]. Disponible en www.guiasalud.es/GPC/GPC_441_ Reflujo_VU.pdf

4. Wennerstrom M, Hansson S, Modal U, Sixt R, Stokland E. Renal function 16 to 26 years after the first urinary tract infection in childhood. Arch Pediatr Adolesc Med. 2000;154:339-45.

5. Marra G, Oppezzo C, Ardissino G. Severe vesioureteral reflux and chronic renal failure: a condition peculiar to male gender? J Pediatr. 2004;144: 677-81.

6. Downs SM. Technical report: urinary tract infections in febrile infants and young children. The urinary tract subcommittee of the American Academy of Pediatrics. Committee on Quality Improvement. Pediatrics. 1999;103;e54.

7. Craig JC, Irwig LM, Knght JF, Roy LP. Does treatment of vesicoureteric reflux in childhood prevent end stage renal disease attributable to reflux nephropathy? Pediatrics. 2000;105:1236-41. 